\title{
Research on Beidou and GPS dual satellite positioning algorithms for substation site positioning
}

\author{
Junjie Feng ${ }^{*}$, Junfeng Shi, Lei Gao, and Hongwei Huang \\ State Grid Maintenance Co. of SEPC, Taiyuan, Shanxi030026, PR China
}

Received: 12 October 2020 / Accepted: 18 November 2020

\begin{abstract}
With the rapid development of global satellite navigation system technology, multi-mode satellite navigation positioning technology that can be compatible with multiple navigation systems at the same time is bound to become the future development trend. Compared with the single-mode navigation system, the multimode navigation system has many advantages, such as a large number of visible stars, a wide coverage, high positioning accuracy, and good reliability. Based on this, this paper applies Beidou and GPS dual-satellite positioning algorithms in substations and studies its key technologies. Specifically, with the aid of MATLAB to solve the Beidou double satellite positioning, GPS positioning results, and the big dipper, GPS double satellite positioning in two times, four times, eight times more weight under the condition of coordinate values and the actual coordinates on the $\mathrm{x}, \mathrm{y}$, and $\mathrm{z}$ axes deviation were compared. The results pointed out that GPS and Beidou double satellite single point positioning effect would be different under different weights. Therefore, in the actual positioning, the positioning effect of the algorithm can be improved by adjusting the weight. In order to test the feasibility of the proposed Beidou and GPS dual satellite positioning algorithms, positioning experiments were carried out, and the results were compared with the single satellite positioning results. The results showed that the curves of Beidou and GPS dual satellite positioning algorithms were highly fitting to the actual curves, confirming the feasibility of the proposed algorithm. This study aims to improve the accuracy and efficiency of substation positioning and navigation for the development of domestic power transformation.
\end{abstract}

Keywords: Substation / multi-mode satellite / Beidou / GPS dual satellite positioning algorithm

\section{Introduction}

With the rapid development of science and technology and the continuous advancement of navigation technology, navigation technology has been widely used in people's daily life and related scientific research activities, especially making important contributions to substation inspection and navigation [1-5]. However, due to the special substation environment and the interference of magnetic fields and other factors, it is difficult for a single-mode positioning and navigation receiver system to efficiently complete the positioning and navigation tasks of the substation [6-8]. At the same time, a single-mode positioning and navigation system will also have problems with fixed errors and the number of satellites, which further inhibits the availability and integrity of the satellite navigation and positioning system $[9,10]$. In order to solve this problem, this paper will propose a solution method for Beidou and GPS dual satellite positioning systems to

\footnotetext{
* Corresponding author: fjyzmn@163.com
}

achieve the dual satellite positioning results and improve the accuracy and efficiency of substation positioning and navigation.

\section{The working principle of Beidou and GPS navigation system}

\subsection{Beidou navigation system}

The Beidou Navigation System is a global navigation and positioning system independently developed in China and compatible with the three existing navigation systems. According to the original intention of the Beidou navigation system, the completed navigation system is mainly committed to providing users with high-quality navigation, positioning, timing, and brief communication services worldwide. At present, it has been widely used in transportation, aviation, military, etc.

The development of the BDS is proceeding steadily on a "regional" and then "global" path. First, the Beidou Navigation Satellite Test System, known as the Beidou 1 
system, was completed in 2000. The GEO operation experiment was carried out to train technical personnel and accumulate experience. Later, the Beidou Regional Navigation Satellite System, or Beidou 2 Regional System for short, was established. More than a dozen satellites, such as geostationary orbit satellites, MEO and IGSO, were launched, and navigation, timing, and positioning services covering the Asia-Pacific region were completed in 2012. Finally, a complete global satellite navigation system is constructed, involving satellites including geostationary and geostationary orbit satellites.

"Beidou 2" consists of three separate parts: a satellite constellation, a ground monitoring station, and user equipment. At present, the Beidou 2 system is comparable to civil GPS in positioning accuracy, which can reach within $10 \mathrm{~m}$, timing accuracy up to $20 \mathrm{~ns}$, and short message delivery of 120 Chinese characters.

\subsection{GPS navigation system}

The Global Position System (GPS) was originally developed by the US Navy as a satellite navigation system to provide Global navigation for arctic nuclear submarines. The system, due to the predecessor of the US military development of a "Meridian", is also known as the "Meridian Satellite System".

The main features of GPS compared with other navigation systems are: GPS system can not only be used for position measurement and navigation, but also for speed and time measurement of moving targets. Among them, GPS time measurement and speed measurement accuracy can reach $0.1 \mathrm{~m} / \mathrm{s}$ and tens of nanoseconds respectively. GPS measurement is not affected by factors such as work place, climate, etc. and can be carried out continuously anytime and anywhere. Similar to the structure of the Beidou navigation system, GPS also is composed of space, ground, and user.

The GPS system's "space constellation" consists of 24 satellites, four of which are evenly distributed within each orbital plane. The distribution of the system's space constellations can effectively ensure that at least four satellites can be observed at any point at any time on the earth at the same time, and the satellite signals will not be greatly changed due to extreme weather in the transmission process.

The "ground monitoring part" of GPS system consists of master control station, monitoring station, and information injection station. "Monitoring station" is a data and information acquisition center under the supervision of the main control station, mainly responsible for satellite status monitoring and information acquisition. On the one hand, the "master control station" coordinates and manages the work of the ground monitoring system. On the other hand, according to the observation data of this station and other stations, various ephemeris data, clock correction parameters and pseudo-range error correction parameters are compiled. The data is then transmitted to the injection station to provide a time reference for the GLOBAL positioning system. Finally, according to the clock information compiled in the navigation message, the satellite deviated from the orbit is adjusted, and the standby satellite is used to replace the failed satellite. The "information injection station" is mainly composed of PC, satellite antenna, and VHF segment transmitter. Under the control of the master control station, all kinds of message data, clock, ephemeris, pseudo-range error parameters and other control instructions are injected into the corresponding satellite storage, and the correctness of the satellite system is tested.

The "user device" of GPS system can be divided into two parts: data receiving and processing. The receiving part is mainly the front-end processing module of various kinds of GPS or GPS-compatible receivers. The data processing part includes all kinds of data processing software and microprocessor. The main function of the receiving module is to receive satellite signals and preliminarily process the signals to obtain some necessary navigation data and measurement information. The processing part refers to the use of various programming tools for subsequent processing of the observed data in order to obtain accurate positioning results.

\section{Systematic observation quantity setting and user position calculation under single system}

\subsection{Systematic observation quantity setting}

As the distance observed between the satellite and the receiver, the pseudo-distance can be measured by measuring the time when the satellite signal is transmitted to the receiver, namely $(r=c \times t)$. In the GPS system, if $t^{j}$ is the moment when the satellite $j$ transmits the signal, $t_{r}$ is the signal reception moment when the local pseudocode generated by the receiver and the satellite signal are cross-correlated to produce the maximum value, and it is assumed that the signal propagates under vacuum and does not consider the influence of errors. Then, the measured pseudorange is the true geometric distance between the satellite and the receiver, which can be expressed as:

$$
R_{r}^{j}=C\left(t_{r}-t^{j}\right)
$$

Where $C$ is the speed of light under vacuum, and the superscript $j$ and the subscript $r$ represent the satellite number and the receiver, respectively.

In the above equation, $t^{j}$ and $t_{r}$ are the actual transmitting and receiving moments of satellite signals. If the effect of the clock difference between the satellite and the receiver is taken into account, the pseudo-distance should be expressed as:

$$
R_{r}^{j}=C\left(t_{r}-t^{j}\right)+C\left(\delta t_{r}-\delta t^{j}\right),
$$

where $\delta t^{j}$ represents the standard clock difference of the time of the satellite clock face relative to the satellite clock. $\delta t_{r}$ is the clock difference of receiver clock face time relative to satellite standard time, and the first term on the right of the above equation can be expressed as:

$$
r_{r}^{j}=C\left(t_{r}-t^{j}\right)=\sqrt{\left(x^{j}-x_{r}\right)^{2}+\left(y^{j}-y_{r}\right)^{2}+\left(z^{j}-z_{r}\right)^{2}}
$$


In equation (3), $r_{r}^{j}$ represents the actual geometric distance between the satellite and the receiver, while $\left(x^{j}, y^{j}, z^{j}\right)$ and $\left(x_{r}, y_{r}, z_{r}\right)$ represent the position coordinates of the $j$-th satellite and receiver $r$ in the same geodetic cartesian coordinate system, respectively.

In the actual pseudo-distance measurement, as satellite signals are affected by ionosphere, troposphere, multipath, relativity, tide and other factors in the propagation process, the real pseudo-distance should be expressed as follows:

$$
R_{r}^{j}=r_{r}^{j}+C\left(\delta t_{r}-\delta t^{j}\right)+\delta_{\text {tro }}+\delta_{i o n}+\delta_{m u l}+\varepsilon,
$$

In equation (4), $\delta_{i o n}$ represents the pseudo-distance error caused by the ionosphere. $\delta_{\text {tro }}$ represents the pseudodistance error caused by the troposphere. $\delta_{m u l}$ represents the pseudo-distance error caused by the multipath effect. $\varepsilon$ represents the pseudo-distance error under the influence of other factors. $C\left(\delta t_{r}-\delta t^{j}\right)$ represents the pseudorange error caused by the clock difference between the receiver and the satellite, and in the above formula, except for the receiver clock difference $\delta t_{r}$, all the error values and satellite position coordinates can be directly or indirectly calculated from the satellite ephemeris file. Therefore, equation (5) can be expressed as a function:

$$
R_{r}^{j}=f\left(x_{r}, y_{r}, z_{r}, \delta t_{r}\right)
$$

Similar to the observation principle of THE GPS navigation system, the Beidou and GPS dual navigation satellite systems can observe the GPS and Beidou satellite ranging at the same time during pseudo-distance observation. Therefore, different time standards (BDT and GPST) can be used according to Beidou and GPS, using the clock difference parameters between the two systems to perform pseudorange fusion. The details are as follows.

Assuming that the receiver of the dual-satellite system takes GPST as the benchmark, when GPS is observed, the propagation time of the satellite signal can be obtained by subtracting the GPST standard time when the receiver receives the signal from the satellite. When the observing satellite is the Beidou star, the propagation time of the signal from the satellite clock face to the receiver is expressed as the GPST standard time when the receiver receives the Beidou satellite signal minus the BDT standard time when the Beidou satellite transmits the signal. The pseudo-range between the Beidou satellite and the receiver can be obtained according to the previous equation:

$$
\begin{aligned}
R_{r-B D}^{k} & =C\left(t_{r-G P S T}-t_{B D T}^{k}\right) \\
& =C\left(t_{r-G P S T}-\left(t_{G P S T}^{k}-\delta t_{G P S T-B D T}\right)\right) \\
& =C\left(t_{r-G P S T}-t_{G P S T}^{k}\right)+C \delta t_{G P S T-B D T},
\end{aligned}
$$

In the above formula, $k$ represents the Beidou satellite number, $R_{r-B D}^{k}$ represents the pseudorange between the $k$-th Beidou satellite and the receiver, $t_{r-G P S T}$ is the GPST time when the receiver receives the signal, and $t_{B D T}^{k}$ is the BDT time when the Beidou satellite transmits the signal. As can be seen from the above equation, the first term on the right of the equals sign is similar to equation (2), indicating the observation pseudo-distance between the receiver and Beidou satellite. The second term is the error term caused by the clock difference between different systems. Therefore, compared with a single system, a new system time difference $\delta t_{G P S T-B D T}$ is introduced in the observation pseudorange of the Beidou satellite in the Beidou and GPS dual satellite systems. Similarly, the expression form of the function to rewrite the above formula into equation (5) is as follows

$$
R_{r-B D}^{k}=f\left(x_{r}, y_{r}, z_{r}, \delta t_{r}, \delta t_{G P S-B D}\right),
$$

Finally, equations (5) and (7) can be used to obtain the pseudo-distance equation between the satellite and the receiver in Beidou and GPS twin satellite systems, which can be expressed as follows:

$$
\left\{\begin{array}{l}
R_{r-G P S}=f\left(x_{r}, y_{r}, z_{r}, \delta t_{r}\right) \\
R_{r-B D}=f\left(x_{r}, y_{r}, z_{r}, \delta t_{r}, \delta t_{G P S-B D}\right)
\end{array} .\right.
$$

\subsection{User position calculation under single system}

The essence of satellite navigation is to calculate the amount of observations. The essence of satellite navigation is to calculate the amount of observations. In A GPS navigation system, the user's position coordinates can be calculated after determining the satellite's position coordinates and the observation point's observation pseudodistance. According to pseudo-distance observation, the pseudo-distance between any satellite and receiver can be expressed as follows:

$$
\begin{aligned}
R_{r}^{j}= & r_{r}^{j}+C\left(\delta t_{r}-\delta t^{j}\right)+\delta_{\text {tro }}+\delta_{\text {ion }}+\delta_{m u l}+\varepsilon \\
= & \sqrt{\left(x^{j}-x_{r}\right)^{2}+\left(y^{j}-y_{r}\right)^{2}+\left(z^{j}-z_{r}\right)^{2}} \\
& +C\left(\delta t_{r}-\delta t^{j}\right)+\delta_{\text {tro }}+\delta_{i o n}+\delta_{m u l}+\varepsilon .
\end{aligned}
$$

For the above equation, the pseudo-distance equation after error correction can be expressed as follows:

$$
\begin{aligned}
\rho^{j} & =\sqrt{\left(x^{j}-x_{r}\right)^{2}+\left(y^{j}-y_{r}\right)^{2}+\left(z^{j}-z_{r}\right)^{2}}+C \delta t_{r} \\
& =f\left(x_{r}, y_{r}, z_{r}\right)+C \delta t_{r}
\end{aligned}
$$

where $\rho^{j}$ represents the pseudo-distance measured value after error correction. As the above equation $f\left(x_{r}, y_{r}, z_{r}\right)+C \delta t_{r}$ is a nonlinear equation, the pseudodistance of at least four satellites is needed when the least square method is used to solve the coordinates, and each set of equations needs to be linearized.

Suppose that the approximate receiver position $\left(x_{r 0}, y_{r 0}, z_{r 0}\right)$ is known, and the deviation between the real receiver position and the approximate receiver position is represented by $\left(\Delta x_{r}, \Delta y_{r}, \Delta z_{r}\right)$, then

$$
\left\{\begin{array}{l}
x_{r}=x_{r 0}+\Delta x_{r} \\
y=y_{r 0}+\Delta y_{r} \\
z_{r}=z_{r 0}+\Delta z_{r}
\end{array} .\right.
$$


Then $f\left(x_{r}, y_{r}, z_{r}\right)$ can be expressed as:

$f\left(x_{r}, y_{r}, z_{r}\right)=f\left(x_{r 0}+\Delta x_{r}, y_{r 0}+\Delta y_{r}, z_{r 0}+\Delta z_{r}\right)$.

For the Taylor expansion of the above equation at point $\left(x_{r 0}, y_{r 0}, z_{r 0}\right)$, the remainder terms after the first partial derivative are ignored, and the following arrangement can be obtained:

$$
\begin{aligned}
& \Delta \rho^{j}=f\left(x_{r 0}, y_{r 0}, z_{r 0}\right)-f\left(x_{r}, y_{r}, z_{r}\right)-C \delta t_{r} \\
& \quad=\frac{x^{j}-x_{r 0}}{r_{r 0}^{j}} \Delta x_{r}+\frac{y^{j}-y_{r 0}}{r_{r 0}^{j}} \Delta y_{r}+\frac{z^{j}-z_{r 0}}{r_{r 0}^{j}} \Delta z_{r}-C \delta t_{r} .
\end{aligned}
$$

In the above equation, $r_{r 0}^{j}$ is the geometric distance between $j$ satellite and receiver approximate position $\left(x_{r 0}, y_{r 0}, z_{r 0}\right)$ :

$$
\begin{gathered}
r_{r 0}^{j}=\sqrt{\left(x^{j}-x_{r 0}\right)^{2}+\left(y^{j}-y_{r 0}\right)^{2}+\left(z^{j}-z_{r 0}\right)^{2}} \\
\text { If } a_{x j}=\frac{x^{j}-x_{r 0}}{r_{r 0}^{j}}, a_{y j}=\frac{y^{j}-y_{r 0}}{r_{r 0}^{j}} \text { and } a_{z j}=\frac{z^{j}-z_{r 0}}{r_{r 0}^{j}}, \text { equation }
\end{gathered}
$$

can be simplified as:

$$
\Delta \rho^{j}=a_{x j} \Delta x_{r}+a_{y j} \Delta y_{r}+a_{z j} \Delta z_{r}-C \Delta t_{r} .
$$

According to the linear equation, the above formula can be written as:

$$
\Delta \rho=A \Delta x .
$$

When the observation satellite is $m$, the above equation can be written as:

$\Delta \rho=\left\lfloor\begin{array}{l}\Delta \rho^{1} \\ \Delta \rho^{2} \\ \vdots \\ \Delta \rho^{m}\end{array}\right\rfloor A=\left[\begin{array}{cccc}a_{x 1} & a_{y 1} & a_{z 1} & 1 \\ a_{x 2} & a_{y 2} & a_{z 2} & 1 \\ \vdots & \vdots & \vdots & \vdots \\ a_{x m} & a_{y m} & a_{z m} & 1\end{array}\right\rfloor \Delta x=\left\lfloor\begin{array}{l}\Delta x_{r} \\ \Delta y_{r} \\ \Delta z_{r} \\ -C \Delta t_{r}\end{array}\right\rfloor$

When $m$ is greater than or equal to 4 , the solution can be obtained according to the least square method:

$$
\Delta x=\left(A^{T} A\right)^{-1} A^{T} \Delta \rho .
$$

At this point, the solution is: $\left(\Delta x_{1}, \Delta y_{1}, \Delta z_{1}\right)$, and the new initial coordinates are obtained by adding this solution to the approximate position of the receiver:

$$
\left\{\begin{array}{l}
x_{r 1}=x_{r 0}+\Delta x_{1} \\
y_{r 1}=y_{r 0}+\Delta y_{1} \\
z_{r 1}=z_{r 0}+\Delta z_{1}
\end{array}\right.
$$

Bring this approximate value back into equation (19) for iteration until the accuracy of $\Delta x_{m}, \Delta y_{m}$, and $\Delta z_{m}$ reaches below the meter level, and finally the position coordinates of the receiver can be obtained.

\section{User location calculation under Beidou and GPS dual satellite systems}

\subsection{Beidou and GPS dual satellite system positioning algorithm}

Based on the single-system positioning principle, this paper uses the pseudo-range observation method to solve the user coordinates under the Beidou and GPS dual satellite systems. The difference is that when performing pseudorange fusion of the combined system, the pseudorange error caused by the time deviation of the system needs to be considered. Therefore, the pseudorange equation of the dual-satellite system will introduce a new time system difference unknown factor, and the combined equation will contain five unknown parameters. the fused pseudorange equation can be expressed as follows:

$$
\left\{\begin{array}{l}
R_{r-G P S}=f\left(x_{r}, y_{r}, z_{r}, \delta t_{r}\right) \\
R_{r-B D}=f\left(x_{r}, y_{r}, z_{r}, \delta t_{r}, \delta t_{G P S-B D}\right)
\end{array} .\right.
$$

It is obtained by the transformation of the single system positioning equation $\Delta \rho=A \Delta x$ after linearization in the previous section.

$$
\Delta \rho-A \Delta x=\Delta \varepsilon,
$$

where $\Delta \varepsilon$ represents the measurement error term. The pseudo-range observation equation of Beidou and GPS combined system can be expressed as:

$$
\left\{\begin{array}{l}
\Delta \rho_{g p s}-A_{g p s} \Delta x=\Delta \varepsilon_{g p s} \\
\Delta \rho_{b d}-B_{b d} \Delta x=\Delta \varepsilon_{b d}
\end{array} .\right.
$$

Assuming that the observed GPS stars are $m$ and Beidou satellites are $n$, the parameters in the above formula are expressed as follows:

$$
\begin{aligned}
\Delta \rho_{g p s}= & {\left[\begin{array}{c}
\Delta \rho_{g p s}^{1} \\
\Delta \rho_{g p s}^{2} \\
\vdots \\
\Delta \rho_{g p s}^{m}
\end{array}\right] A_{g p s}=\left[\begin{array}{ccccc}
a_{x 1} & a_{y 1} & a_{z 1} & 1 & 0 \\
a_{x 2} & a_{y 2} & a_{z 2} & 1 & 0 \\
\vdots & \vdots & \vdots & \vdots & \vdots \\
a_{x m} & a_{y m} & a_{z m} & 1 & 0
\end{array}\right] } \\
\Delta \varepsilon_{g p s}= & {\left[\begin{array}{c}
\Delta \varepsilon_{g p s 1} \\
\Delta \varepsilon_{g p s 2} \\
\vdots \\
\Delta \varepsilon_{g p s m}
\end{array}\right], } \\
\Delta \rho_{b d}= & {\left[\begin{array}{c}
\Delta \rho_{b d}^{1} \\
\Delta \rho_{b d}^{2} \\
\vdots \\
\Delta \rho_{b d}^{n}
\end{array}\right] B_{b d}=\left[\begin{array}{ccccc}
b_{x 1} & b_{y 1} & b_{z 1} & 1 & 1 \\
b_{x 2} & b_{y 2} & b_{z 2} & 1 & 1 \\
\vdots & \vdots & \vdots & \vdots & \vdots \\
b_{x m} & b_{y m} & b_{z m} & 1 & 1
\end{array}\right] } \\
&
\end{aligned}
$$




$$
\Delta x=\left[\begin{array}{c}
\Delta x_{r} \\
\Delta y_{r} \\
\Delta z_{r} \\
-C \Delta t_{r} \\
-C \Delta t_{G P S-B D}
\end{array}\right] .
$$

According to the pseudo range fusion, the combined observation equations are as follows:

$$
\left[\begin{array}{l}
\Delta \rho_{g p s} \\
\Delta \rho_{b d}
\end{array}\right]-\left[\begin{array}{c}
A_{g p s} \\
B_{b d}
\end{array}\right] \Delta x=\left[\begin{array}{l}
\Delta \varepsilon_{g p s} \\
\Delta \varepsilon_{b d}
\end{array}\right]
$$

For solving the above linear observation equation, the most basic method is to use the least square method to solve it. The solution of the least square method minimizes the sum of squares of the difference between each function value and the actual measured value, so it is optimal. However, since the above equation is composed of two different systems after pseudo-distance fusion, the factor of different positioning accuracy of each system should be taken into account when solving the equation, so that specific weights can be set for different systems. Based on the above considerations, this article uses the adjustment method to solve the positioning based on the least square method. As an effective algorithm for dealing with linear equations, the adjustment method introduces the weight matrix on the basis of the least square method to make it become a full rank linear equation problem. This method has good applicability and finally minimizes the variance between the measured result and the estimated predicted value.

For equation (26), the formula is further rewritten as:

$$
\Delta \rho_{c o m}-A_{c o m} \Delta x=\Delta \varepsilon_{c o m}, P,
$$

where $\Delta \rho_{\text {com }}$ is the $\mathrm{N}$-dimensional pseudo range observation, $A_{\text {com }}$ is the coefficient matrix $(N \times 5)$ dimension in the combined observation equation, $\Delta \varepsilon_{\text {com }}$ is the $\mathrm{N}$-dimensional observation error vector, $\Delta x$ is a 5 -dimensional unknown vector, and $P$ is the symmetric weight matrix in $\mathrm{N}$ by $\mathrm{N}$ dimensions. Among them, $(N=m+n)$ solves the observation equations of the above equation, according to the least squares criterion, there is:

$$
\left(\Delta \varepsilon_{c o m}\right)^{T} P \Delta \varepsilon_{c o m}=\min .
$$

When observed visible star combination $(N=5)$, the least square adjustment is used to solve the equation to get

$$
\Delta x=A_{c o m}^{-1} \Delta \rho_{c o m} .
$$

When observed visible star combination $(N>5)$, the least square adjustment is used to solve the equation to get

$$
\Delta x=\left(A_{c o m}^{T} P A_{c o m}\right)^{-1}\left(A_{c o m}^{T} P \Delta \rho_{c o m}\right) .
$$

\subsection{Weight setting based on least square method}

The weighted least square method is often used in data processing, which requires less prior information for data processing and has better adaptability. The criterion for data optimization is that the variance between the measured data and the predicted value of the estimation model is the smallest after parameter estimation. As the setting of the weight will affect the final positioning result, it is an important factor to be considered in the process of solving the dual satellite positioning. The selection methods of the weight ratio are very diverse, mainly considering the ranging error and other factors of the receiver. In the practical application process, there is a certain connection with factors such as the location of the receiver. Under the ideal condition, by using the same set of original observation data values and using MATLAB to solve the positioning results of Beidou and GPS dual satellite positioning, the $3 \mathrm{D}$ coordinates in the designated coordinate system can be obtained. After the comparison of the true values of the same position, the errors along the $\mathrm{X}, \mathrm{Y}$ and $\mathrm{Z}$ axes can be obtained. The deviation between the coordinate values of Beidou and GPS under the conditions of $2 \mathrm{x}, 4 \mathrm{x}$, and $8 \mathrm{x}$ weight and the actual coordinate values along the $\mathrm{X}, \mathrm{Y}$, and $\mathrm{Z}$ axes is analyzed, as shown in Figures 1-3.

In the process of GPS and Beidou satellite positioning, the influence of weight on positioning results should be considered. It can be seen from the above data errors that the positioning errors of two times weight and four times weight are not significantly different, but both are slightly higher than the accuracy of eight times weight, among which the positioning result of four times weight is the best. The selection of different weights has a different influence on the positioning deviation of GPS and Beidou twin satellites single point positioning; therefore, it is concluded that the selection of appropriate weights can appropriately improve the positioning accuracy of the two satellites in the process of positioning the two satellites.

\section{Positioning result analysis}

In order to further test the feasibility of the Beidou and GPS dual-satellite positioning algorithms proposed in this study, the positioning effects of the single Beidou positioning system and the single GS positioning system were compared, and the results obtained are shown in Figure 4.

It can be seen that although the positioning results of the single Beidou positioning system and the single GPS positioning system are relatively good, there is still a gap compared with the Beidou and GPS dual satellite positioning results. The Beidou and GPS positioning result curves basically fit the actual positioning curve. Therefore, the feasibility of Beidou and GPS positioning systems for substation positioning is confirmed.

\section{Conclusion}

In general, this article first explains the working principles of Beidou and GPS navigation systems and clarifies the respective characteristics of Beidou positioning and navigation systems and GPS positioning and navigation 

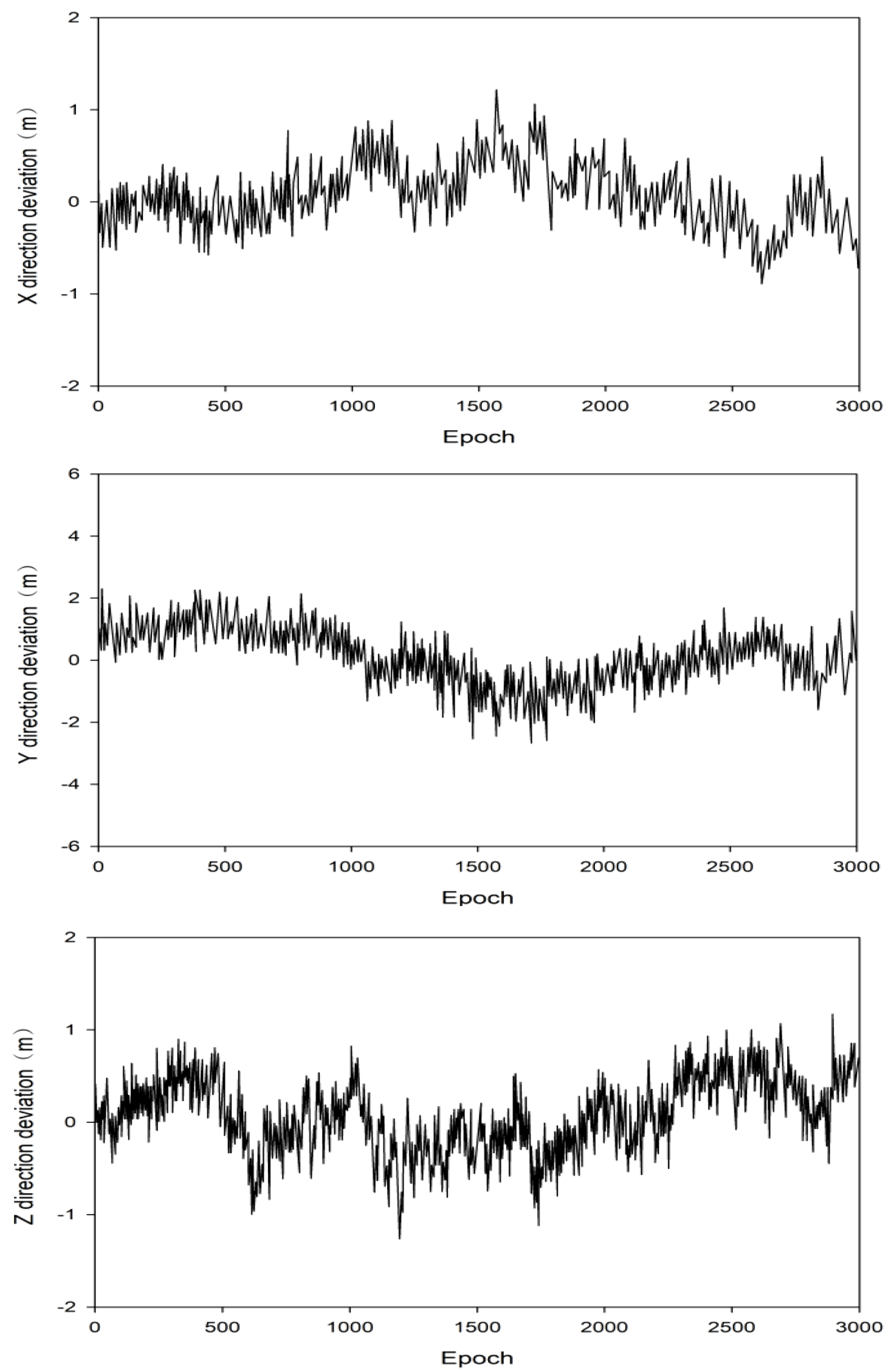

Fig. 1. Two times the power Beidou-GPS dual satellite single point positioning deviation. 

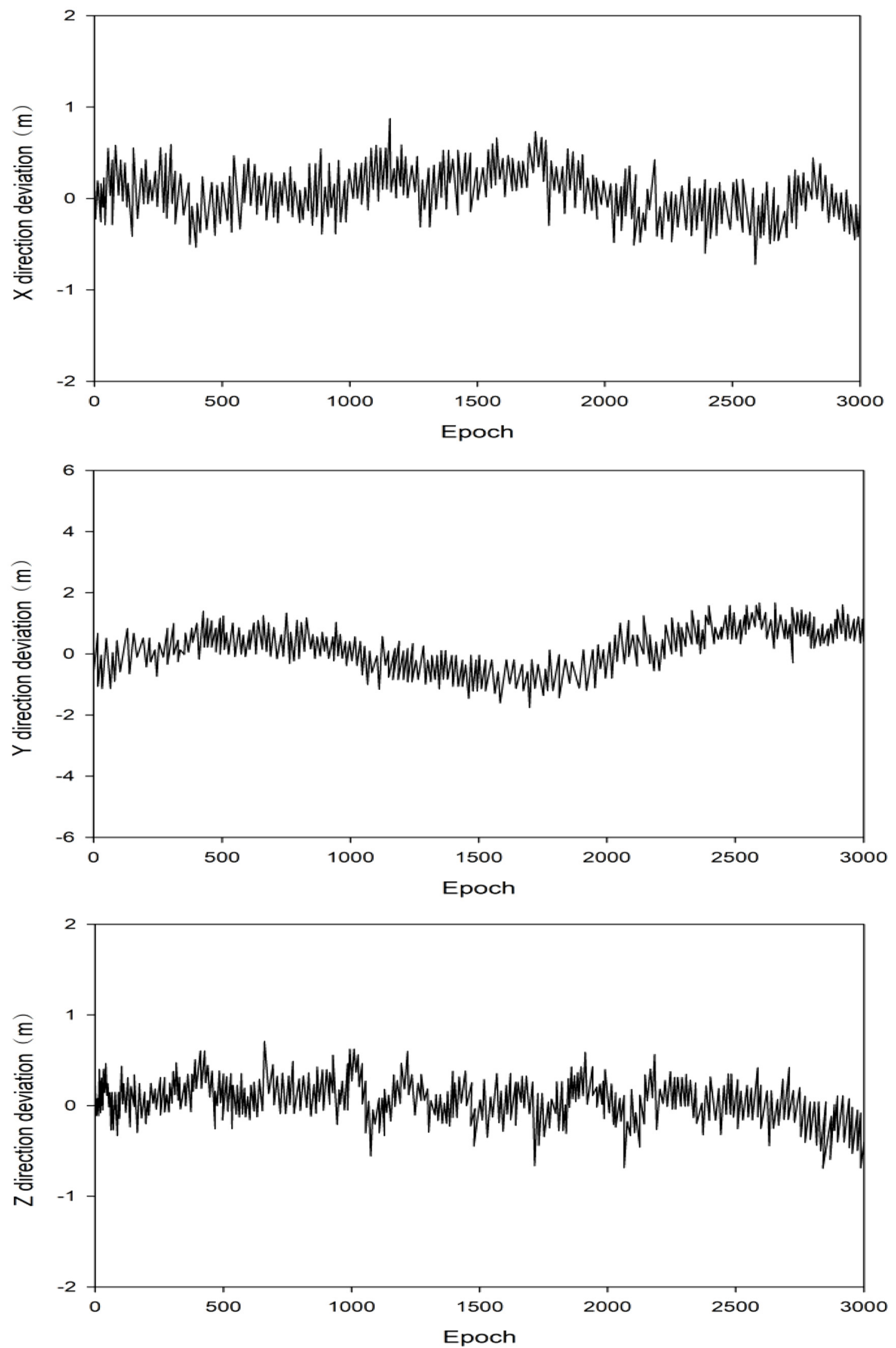

Fig. 2. Four times the power Beidou-GPS dual satellite single point positioning deviation. 

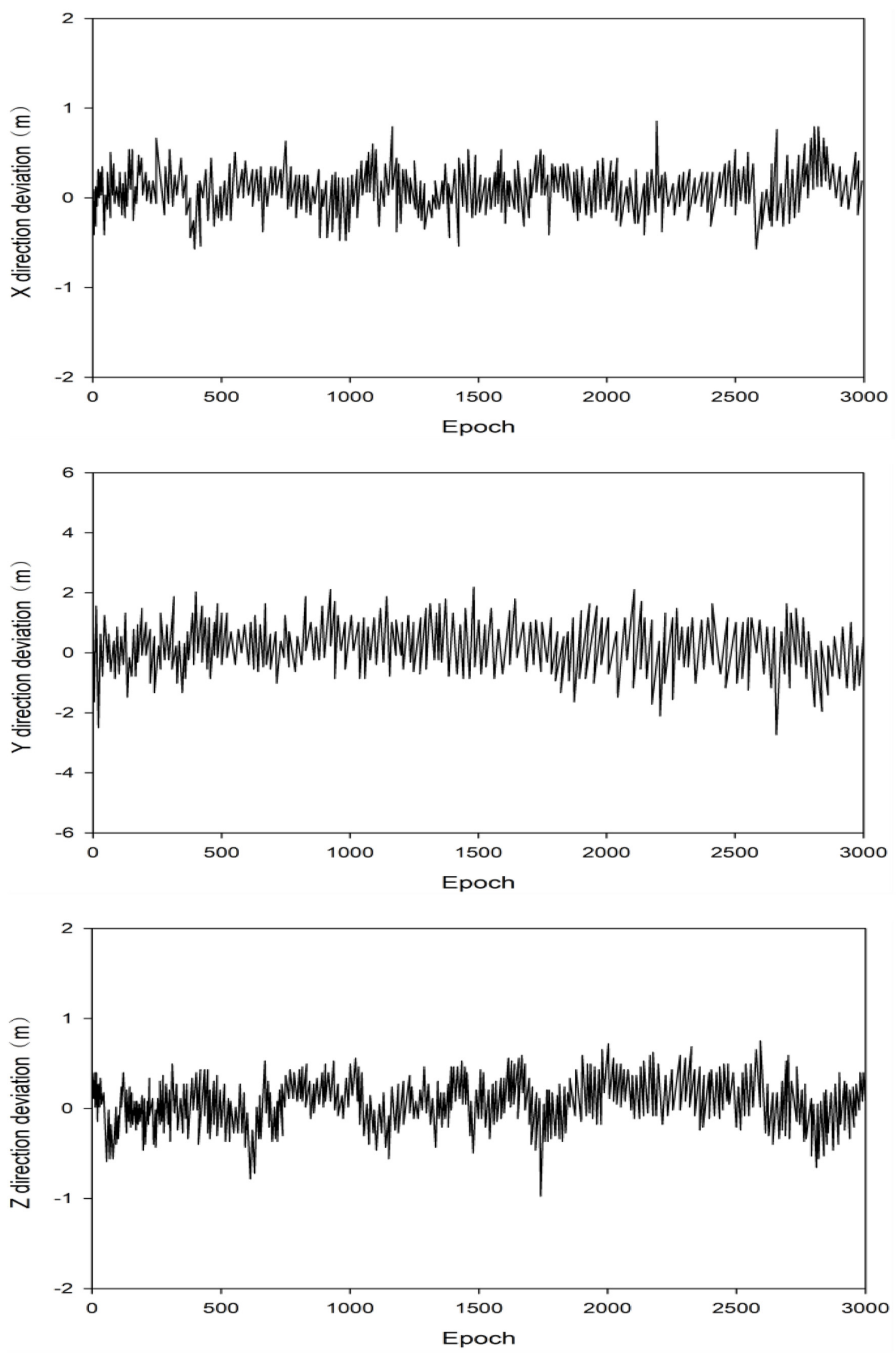

Fig. 3. Eight times the power Beidou-GPS dual satellite single point positioning deviation. 


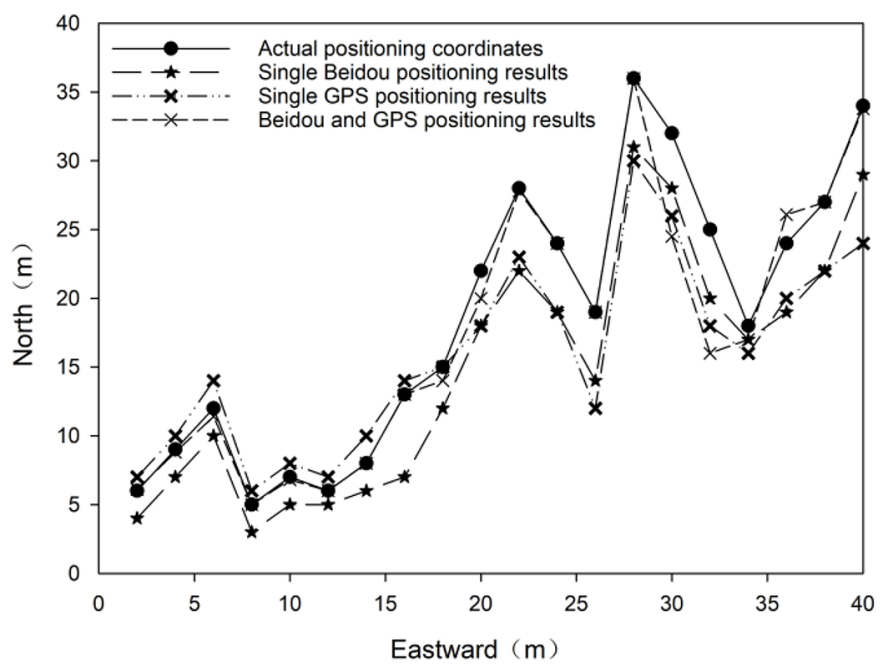

Fig. 4. Comparison of positioning effects under different positioning algorithms.

systems. Then the system observations are set accordingly, and the user position under the single system is calculated. Secondly, on the basis of the previous theoretical analysis, the positioning algorithm of Beidou and GPS dual satellite systems is explained, and its weights are set on the basis of the least square method, and different weights are obtained. For GPS and Beidou dual satellites, the influence of the positioning deviation of the single-point positioning is different; therefore, it is concluded that the accuracy of the dual-satellite positioning can be appropriately improved by selecting the appropriate weight in the dual-satellite positioning process. Finally, the positioning effect of the Beidou and GPS dual-satellite positioning algorithm is compared with the single Beidou positioning algorithm and the single GPS positioning algorithm. The obtained positioning curve has a higher degree of fit with the actual positioning curve, which confirms the feasibility of the application of the dual-satellite positioning algorithm proposed in this study in substations.

\section{References}

1. Y. Jiang, Z. Liu, M.J. Zhang, X.G. Shi, Y. Wang, Electr. Eng. Tech. 22, 126-127+142 (2019)

2. X.Y. Peng, L. Jin, R. Wang, L. Yi, G.Q. Chen, High Voltage Electr. Apparat. 55, 223-232 (2019)

3. X.Y. Xu, R. Yu, Z.P. Xu, Y.F. Hao, Inform. Tech. 43, 71-74 $+78(2019)$

4. Z. Sun, J.L. Hu, J.J. Luo, Y.Y. Li, Autom. Tech. Appl. 37, 82-85 (2018)

5. W. Wang, W. Chen, Q.X. Lu, Appl. Autom. 04, 95-99 (2019)

6. Z.H. Li, Q.H. Fu, W.H. Yu et al., Electr. World 12, 135-136 (2018)

7. J.M. He, J.L. Liu, K. Mao, R.J. Li, Inner Mongolia Electr. Power Tech. 35, 26-28+32 (2017)

8. Z.Y. Wu, Small Medium-sized Enterprise Manag. Tech. (Mid-term J.) 03, 110-111 (2017)

9. J.Q. Han, P.C. Li, H. Jiang, Chem. Ind. Manag. 05, 194 (2017)

10. X. Su, Y.P. Ding, L. Dang, New Tech. New Process 11, 73-75 (2016) 